\title{
THE CLOSED FRIEDMAN WORLD MODEL WITH THE INITIAL AND FINAL SINGULARITIES AS A NON-COMMUTATIVE SPACE
}

\author{
MICHAEL HELLER \\ Vatican Observatory \\ V-12000 Vatican City State* \\ WIESŁAW SASIN \\ Institute of Mathematics, Warsaw University of Technology \\ Plac Politechniki 1, 00-661 Warsaw, Poland
}

\begin{abstract}
The most elegant definition of singularities in general relativity as b-boundary points, when applied to the closed Friedman world model, leads to the disastrous situation: both the initial and final singularities form the single point of the b-boundary which is not Hausdorff separated from the rest of space-time. We apply Alain Connes' method of non-commutative geometry, defined in terms of a $C^{*}$-algebra, to this case. It turns out that both the initial and final singularities can be analysed as representations of the $C^{*}$-algebra in a Hilbert space. The method does not distinguish points in space-time, but identifies space slices of the closed Friedman model as states of the corresponding $C^{*}$-algebra.
\end{abstract}

1. Introduction. There is growing both observational and theoretical evidence that the Universe in its history went through a superdense phase commonly called Big Bang. According to classical theorems on the existence of singularities (see [7]), in the Big Bang the histories of all non-zero rest-mass particles and photons broke down (or rather suddenly appeared out of non-existence). In the nowadays prevalent view, such a disaster will be prevented by the future theory of quantum gravity. This theory will, when finally discovered, violate at least one of the conditions (probably the one called the energy condition) of the singularity theorems, and in this way free relativistic physics from the nightmare of singularities. However, there are at least two major reasons for further investigating classical singularities. First, because they constitute a very interesting and rich

1991 Mathematics Subject Classification: 83C47, 81R50.

Key words and phrases: Friedman cosmology, singularities, non-commutative geometry.

*Correspondence address: ul. Powstańców Warszawy 13/94, 33-110 Tarnów, Poland.

The paper is in final form and no version of it will be published elsewhere. 
in consequences study case for differential geometers and, second, because such studies could suggest fruitful ideas in our search for the correct theory of quantum gravity. The present paper is motivated by both these reasons.

Till now, all attempts to mathematically describe the structure of strong curvature singularities, such as the initial and final singularities in the closed Friedman world model, failed, and one had to use rather heuristic criteria (timelike or null geodesic incompleteness) to prove the existence of singularities in some more general situations. Moreover, it has turned out that the best available definition of singularities as points of the bboundary of space-time [20], when applied to the closed Friedman world model leads to the disastrous situation: both the initial and final singularities form the single point of the b-boundary which is not Hausdorff separated from the rest of space-time [2], [13].

To deal with similar situations Alain Connes [4] has developed the theory of noncommutative geometry. The aim of the present paper is to apply this method to the closed Friedman model with its singularities (understood as the b-boundary) and to study the structure of these singularities. Although the work is at its preliminary phase, the results seem to be interesting.

The organization of our material is the following. In section 2 , we briefly summarize Schmidt's construction of the space-time b-boundary and discuss difficulties in which it is involved. In section 3, we demonstrate how the ordinary smooth manifold can be presented as a non-commutative space. This will give us a pedagogical introduction to some ideas of non-commutative geometry and prepare a method which, after a suitable generalization, will be applied, in section 4 , to the closed Friedman model with its singularities.

2. Singular space-times. Let us briefly summarize Schmidt's construction [20] of the b-boundary of space-time. Let $M$ be a smooth space-time manifold carrying a Lorentz metric, and $O M$ the connected component of the orthonormal frame bundle over $M$, $\pi: O M \rightarrow M$. Levi-Civita connection in $M$ determines the family of Riemann (positive definite) metrics on $O M$; all metrics belonging to this family are uniformly equivalent. We choose one of them, by using it we define the distance function $\rho$ on $M$, and construct, in the usual way, the Cauchy completion $\overline{O M}$ of $O M$. Let us notice that the construction of the Cauchy boundary $\partial_{C} O M:=\overline{O M} \backslash O M$ does not depend on the particular choice of the Riemann metric on $O M$ (from the above mentioned family of metrics). The Lorentz group $S O(3,1)$ acts on $O M$ on the right, and since it maps Cauchy sequences into Cauchy sequences one can uniquely extend the action of $S O(3,1)$ to $\overline{O M}$. The quotient space $\bar{M}:=\overline{O M} / S O(3,1)$ is called the $b$-completion of space-time $M$, and $\partial_{b} M:=\bar{M} \backslash M$ the b-boundary of $M . M$ is open and dense in $\bar{M}$.

The distance function $\rho$ on $O M$ can be naturally extended to $\overline{O M}$. If $p=\left\{p_{n}\right\}$ and $q=\left\{q_{n}\right\}$ are Cauchy sequences in $O M$, the distance $\bar{\rho}(p, q)$ between $p$ and $q, p, q \in$ $\partial_{C} O M$, is defined as $\lim _{n \rightarrow \infty} \rho\left(p_{n}, q_{n}\right)$. The distance function $\bar{\rho}$ defines the topology on $\overline{O M}$. Since it is the metric topology it is always Hausdorff. The extended projection $\bar{\pi}: \overline{O M} \rightarrow \bar{M}$, assigning points in $\overline{O M}$ to their equivalence classes under the action of $S O(3,1)$ (or identifying points in $\bar{M}$ with the orbits of $S O(3,1)$ ), induces topology on $\bar{M}$ from that on $\overline{O M}$. However the induced topology need not be metric (since $\bar{\rho}$ is not 
invariant under the action of $S O(3,1))$. This is the source of a non-Hausdorff behaviour in some cases.

Let us notice the strategy underlying this construction. Singularities do not belong to space-time but only to its boundary. However, the singularities are accessible to the investigation from the inside of space-time (i. e., they are defined entirely in terms of space-time domains). This nicely corresponds to the current cosmological practice which tries to reconstruct the structure of the beginning from the later history of the Universe, but from the geometric point of view one would perhaps prefer to deal with space-time together with its singularities regarded as a single object.

Schmidt's construction is very elegant, but to compute b-boundaries for particular cases turned out to be a difficult task. In the original Schmidt's paper only rather toy-models were computed, and in these cases the construction worked well. In 1976 Bosshard [2] and in 1977 Johnson [13] succeeded to show that in the closed Friedman model the initial and final singularities form the single point of the b-boundary, and that the topology of the b-completed space-times of both closed Friedman solution and Schwarzshild solution is not Hausdorff. This discovery practically eliminated Schmidt's construction as a working definition of singularities.

In our previous works [12], [19], [9], we have elucidated the nature of difficulties in which Schmidt's construction is involved. Let us briefly discuss this problem.

In 1960 Koszul [14] developed an algebraic approach to differential geometry in terms of a (commutative) associative algebra $\mathcal{C}$ and $\mathcal{C}$-modules. In the case of a smooth manifold $M, \mathcal{C}$ is the algebra of smooth functions on $M$, and $\mathcal{C}$-modules are spaces of smooth sections of smooth vector bundles over M. Subsequently, many authors tried various modifications of this approach (for review see [8]). The general idea was to consider any algebra of functions (perhaps with some additional conditions) as smooth functions from definition, and accordingly develop differential geometry. In this spirit, we have recently developed the theory of structured spaces and applied it to investigate b-completed spacetimes [11].

By a structured space we understand the pair $(M, \mathcal{C})$, where $M$ is a topological space and $\mathcal{C}$ a sheaf of algebras of real functions on $M$ such that for any open set $U \subset M$ and any functions $\omega \in \mathbf{R}, f_{1}, \ldots, f_{n} \in \mathcal{C}(U)$, for any $n \in \mathbf{N}$, one has $\omega \circ\left(f_{1}, \ldots, f_{n}\right) \in \mathcal{C}(U)$. It is a strong generalization of the usual manifold concept, and it works well when it is applied to space-times with various types of singularities [18], [6], [11]. Let us suppose that $(M, \mathcal{C})$ is a structured space equivalent to the space-time manifold of the closed Friedman world model. We have proved that there exists the unique differential structure $\overline{\mathcal{C}}$ which is a prolongation of $\mathcal{C}$ to the b-completed space-time $\bar{M}$ (i.e., such that $\overline{\mathcal{C}} \mid M=\mathcal{C}$ ), and that $\overline{\mathcal{C}}$ consists only of constant functions on $\bar{M}$ [9], [11]. The topology on $\bar{M}$, as defined by the family of constant functions, is evidently non-Hausdorff. Moreover, since the derivations of constant functions vanish the "bundle length" of any curve joining the initial and final singularities is zero, and consequently both these singularities form the same point of the b-boundary. We have also shown that such situations occur always when the fiber $\bar{\pi}^{-1}\left(x_{0}\right)$ over the singular point $x_{0} \in \partial_{b} M$ degenerates to the single point [9], [10]. We call such singularities malicious singularities. The central Schwarzschild singularity belongs to this type. 
Let $\bar{M}$ be a space-time with a malicious singularity. Since the differential structure $\mathcal{C}$ is a sheaf of function algebras we can always work with $\mathcal{C}(U)$, where $U$ is open subset of $M$, or even with $\mathcal{C}(M)$ ( since $M$ is open in $\bar{M}$ ), without any problems, but we cannot extend our geometric structures to $\bar{M}$ because then the topological problems begin. In this sense, malicious singularities remain non-accessible. To deal with them we need further generalization of geometry. It is provided by changing from commutative to noncommutative algebras.

3. Non-commutative geometry: a simple example. As is well known, any topological manifold $M$ can be reconstructed by suitably gluing together open subsets of some number of copies of Euclidean spaces, but for simplicity let us assume that $M$ is compact. Let $N$ be a disjoint union of such open subsets, $N=\bigcup U_{j}$. We define the equivalence relation $R \subset N \times N$ in the following way: for $x, y \in N, x R x$ iff $p(x)=p(y)$ where $p: N \rightarrow M$ is a suitably defined surjection. ${ }^{1}$ Then $M=N / R$ (for details see [1, p. 31]).

Now, following Connes [4, p. 86], let us define the graph of the above equivalence relation

$$
\mathcal{R}=\{(x, y) \in N \times N: x R y\}
$$

and endow it with the local compact topology. Let further $C_{0}(\mathcal{R})$ be the vector space of continuous functions on $\mathcal{R}$ vanishing at infinity. It is easy to show that $C_{0}(\mathcal{R})$ with the operations of convolution and involution defined in the following way

$$
\begin{gathered}
(f * g)(x, z)=\sum_{x R y R z} f(x, y) g(y, z) \\
f^{*}(x, y)=\overline{f(x, y)}
\end{gathered}
$$

is a $C^{*}$-algebra which we shall denote by $C^{*}(\mathcal{R})$.

It can be shown $[4$, pp. 87 and 153$]$ that the algebra $C^{*}(\mathcal{R})$ coincides with the $C^{*}$ algebra of compact endomorphisms of the continuous field of Hilbert spaces $\left(\mathcal{H}_{x}\right)_{x \in M}$ over $M$, where $\mathcal{H}_{x}=l^{2}\left(p^{-1}(x)\right)$ for every $x \in M$. As is well known, any topological manifold $M$ can be defined in terms of the commutative algebra $C(M)$ of continuous (complex) functions on $M$ (this definition is equivalent to the one in terms of local maps). We thus have two descriptions of the same manifold: (i) in terms of the non-commutative $C^{*}$-algebra $C^{*}(\mathcal{R})$, and (ii) in terms of the commutative $C^{*}$-algebra $C(M)$. These two algebras are strongly Morita equivalent: $C^{*}(\mathcal{R}) \simeq C(M)$. (Let us remind that strong Morita equivalence plays the role of an isomorphism for $C^{*}$-algebras; two strongly Morita equivalent commutative algebras are isomorphic in the usual sense; see [4, pp. 152-159], [15, pp. 140-141].) The main difference between these two descriptions is that the algebra $C(M)$ distinguishes points of $M$, whereas the algebra $C^{*}(\mathcal{R})$ does not. Information about points is somehow encoded in the family of Hilbert spaces $\left(\mathcal{H}_{x}\right)_{x \in M}$ which is parametrized by points of $M$. But both these algebras lead to the same topological invariants, in particular to the same K-theory. The main advantage of the approach in terms of the

\footnotetext{
${ }^{1}$ In other words, $x R y$ iff there exist coordinate maps $\phi_{i}: U_{i} \rightarrow \mathbf{R}^{n}$ and $\phi_{j}: U_{j} \rightarrow \mathbf{R}^{n}$, such that $U_{i} \cap U_{j} \neq \emptyset$, and $x=\left(\phi_{i} \circ \phi_{j}^{-1}\right)(y)$.
} 
$C^{*}$-algebra $C^{*}(\mathcal{R})$ is that in strongly singular situations, when the traditional approach fails, it remains valid. In such situations one could truly speak of the desingularization method with the help of a $C^{*}$-algebra. The above simple example illustrates the main features of this method. The essential points are: 1 ) To change from the space $N / R$ to a suitably organized subset $\mathcal{R}$ of $N \times N$; we shall call it "pairing process". In our case $N / R$ was a manifold, but the fact that it was given as a quotient set suggests that, in general, this space might have some pathologies. 2) To define a suitable $C^{*}$-algebra on $\mathcal{R}$. 3) To extract information on $N / R$ from this $C^{*}$-algebra. In the next section we shall use this approach to study space-time with malicious singularities.

4. Desingularization procedure. Let us start with a space-time $\bar{M}=\overline{O M} / S O(3,1)$ with one or more malicious singularities understood as b-boundary points. For the sake of concreteness we could think about the closed Friedman world model with its initial and final singularities.

To perform the "pairing process" let us notice that since the structure group $S O(3,1)$ acts along fibres on the right, the transformation from a frame $p$ to a frame $q$ (both frames in the same fibre) can be presented as a pair $\gamma:=(p, p g)$, where $q=p g$, and a single frame as the pair $(p, p e)$ where $e$ is the unit of $S O(3,1)$. One can regard $(p, p g)$ as an arrow beginning at $p$ and ending at $p g$, and $(p, p e)$ as a loop at $p$. We shall treat these pairs or arrows as "elementary units" of our construction. We can also write simply $(p, g)$ and $(p, e)$, respectively.

Now, we define two sets

$$
G=\overline{O M} \times S O(3,1), \quad G^{(0)}=\overline{O M} \times\{e\}=\{(p, e): p \in \bar{M}\}
$$

(notation is the same as in section 2; a frame in a singular fibre should be understood as a limit of equivalence class of Cauchy sequences) and two mappings

$$
r, s: G \rightarrow G^{(0)}
$$

such that $r(p, g)=p$ and $s(p, g)=p g$. Now, we can introduce the composition of pairs

$$
\circ: G^{(2)} \rightarrow G
$$

by

$$
\left(p, g_{1}\right)\left(q, g_{2}\right)=\left(p, g_{1} g_{2}\right)
$$

where

$$
\begin{aligned}
G^{(2)}:= & \left\{\left(\gamma_{1}, \gamma_{2}\right) \in O M \times O M: s\left(\gamma_{1}\right)=r\left(\gamma_{2}\right)\right\} \\
= & \left\{\left(\left(p, g_{1}\right),\left(q, g_{2}\right)\right): p g_{1}=q\right\} .
\end{aligned}
$$

We have also the inverse $(p, g)^{-1}$ of $(p, g)$ which is equal to $\left(p g, g^{-1}\right)$.

It is straightforward to verify that in this way we have defined a groupoid structure on $G=\overline{O M} \times S O(3,1)$. This groupoid will be called the groupoid of orthonormal frames over $\bar{M} .^{2}$

\footnotetext{
${ }^{2}$ This construction is quite general. In fact, the construction of the preceding section could also be carried out in terms of a groupoid: graph of any equivalence relation can be given the groupoid structure (see [17, p. 7]).
} 
The groupoid $G$ of orthonormal frames over $M$ is smooth as a structured space: the differential structure on $\overline{O M}$ can be pulled back from the Euclidean space into which $\overline{O M}$ is embedded and $S O(3,1)$ itself carries a smooth manifold structure.

In the following, two sets will be important: the set of arrows which begin at $p \in \overline{O M}$,

$$
G^{p}=\{(p, g): g \in S O(3,1)\},
$$

and the set of arrows which end at $q$,

$$
G_{q}=\left\{\left(q g^{-1}, q\right): g \in S O(3,1)\right\} .
$$

It is easy to see that both these sets have the structure of the smooth group manifold $S O(3,1)$, and that this structure is preserved even if $p$ and $q$ are in the fibre over a malicious singularity. This is an important step in the desingularization procedure.

To define a suitable $C^{*}$-algebra we shall strictly follow Connes' method [4, pp. 99-102]. We construct the line bundle $\tau: \Omega^{1 / 2} \rightarrow \bar{G}$ over the groupoid of orthonormal frames, where

$$
\Omega^{1 / 2}=\bigcup_{\gamma \in G} \Omega_{\gamma}^{1 / 2}
$$

and $\Omega_{\gamma}^{1 / 2}$ is the set of mappings

$$
\begin{gathered}
\Omega_{\gamma}^{1 / 2}=\left\{\rho: \bigwedge^{k} T_{\gamma}\left(G^{p}\right) \otimes \bigwedge^{k} T_{\gamma}\left(G_{q}\right) \rightarrow \mathbf{C}:\right. \\
\left.\rho(\lambda \nu)=|\lambda|^{1 / 2} \rho(\nu), \lambda \in \mathbf{R}, \nu \in \bigwedge^{k} T_{\gamma}\left(G^{p}\right) \otimes \bigwedge^{k} T_{\gamma}\left(G_{q}\right)\right\}
\end{gathered}
$$

for $\gamma=(p, p g), \operatorname{dim}\left(G^{p}\right)=\operatorname{dim}\left(G_{q}\right)=6$. It can be shown that this line bundle is trivial $\Omega^{1 / 2}=G \times \tilde{\Omega}^{1 / 2}$ where

$$
\tilde{\Omega}^{1 / 2}=\left\{\rho: \bigwedge^{6} o(3,1) \otimes \bigwedge^{6} o(3,1) \rightarrow \mathbf{C}\right\}
$$

By $s o(3,1)$ we have denoted the Lie algebra of the Lie group $S O(3,1)$. To show that it is indeed a trivial bundle we define the bijection $F: \Omega^{1 / 2} \rightarrow \bar{G} \times \tilde{\Omega}^{1 / 2}$ by $F(\rho)=(\gamma, \hat{\rho})$, where

is defined by

$$
\hat{\rho}: \bigwedge^{k} o(3,1) \otimes \bigwedge^{k} o(3,1) \rightarrow \mathbf{C}
$$

$$
\begin{gathered}
\hat{\rho}\left(u_{1} \wedge \ldots \wedge u_{k} \otimes v_{1} \wedge \ldots \wedge v_{k}\right)= \\
=\rho\left(\iota_{*}^{p} u_{1} \wedge \ldots \wedge \iota_{*}^{p} u_{k} \otimes \iota_{q *} v_{1} \wedge \ldots \wedge \iota_{q *} v_{k}\right),
\end{gathered}
$$

and the mappings

$$
\begin{gathered}
\iota^{p}: O(3,1) \rightarrow\{p\} \times O(3,1)=G^{p}, \\
\iota_{q}: O(3,1) \rightarrow\left\{q g^{-1}\right\} \times O(3,1)=G^{q}
\end{gathered}
$$

are given by

$$
\begin{gathered}
\iota^{p}(h)=(p, h g), \\
\iota_{q}(h)=\left(q g^{-1}, h g\right),
\end{gathered}
$$

with $h \in O(3,1)$; the last two equalities define diffeomorphisms between manifolds. This trivial structure of the line bundle is preserved at singularities (even if they are malicious). 
To define the required $C^{*}$-algebra we first change the space of smooth compactly supported cross-sections $C_{c}^{\infty}\left(\bar{G}, \Omega^{1 / 2}\right)$ of the line bundle $\tau: \Omega^{1 / 2} \rightarrow \bar{G}$ into an involutive algebra by defining the convolution (as multiplication in the algebra)

$$
(s * t)(\gamma)=\int_{G^{p}} s\left(\gamma_{1}\right) t\left(\gamma_{2}\right)
$$

where $s, t \in C_{c}^{\infty}\left(\bar{G}, \Omega^{1 / 2}\right), \gamma=(p, g), \gamma=\gamma_{1} \circ \gamma_{2}$, and the involution $s \mapsto s^{*}$ as $s^{*}(\gamma)=$ $\overline{s\left(\gamma^{-1}\right)}$.

The final step consists in proving [3], [17] that for each $q \in G^{(0)}$ the expression

$$
\left(\pi_{q}(s) \xi\right) \gamma=\int_{G_{q}} s\left(\gamma_{1}\right) \xi\left(\gamma_{1}^{-1} \gamma\right)
$$

where $\gamma \in G_{q}, \xi \in L^{2}\left(G_{q}\right), s \in C_{c}^{\infty}\left(\bar{G}, \Omega^{1 / 2}\right)$, defines an involutive non-degenerate representation

$$
\pi_{q}: C_{c}^{\infty}\left(\bar{G}, \Omega^{1 / 2}\right) \rightarrow \operatorname{End} L^{2}\left(G_{q}\right)
$$

of $C_{c}^{\infty}\left(\bar{G}, \Omega^{1 / 2}\right)$ in the Hilbert space $L^{2}\left(G_{q}\right)$ of the square integrable functions on the group manifold $G_{q}$. The completion of $C_{c}^{\infty}\left(\bar{G}, \Omega^{1 / 2}\right)$ with respect to the norm

$$
\|s\|=\sup _{q \in G^{(0)}}\left\|\pi_{q}(s)\right\|
$$

is a $C^{*}$-algebra. We shall denote it by $C^{*}(\overline{O M})$. This algebra contains information about space-time with singularities. Our next task is to decipher this information.

5. Discussion. The algebra $C^{*}(\overline{O M})$ is a non-commutative algebra, and it does not identify points. Space-time with malicious singularities is non-Hausdorff and can be treated only globally. If we want to recover points (and their neighborhoods) we must change to a commutative (functional) algebra over the regular (non-singular) part of space-time but then, of course, we loose information about singularities.

Let us denote $C^{*}(\overline{O M})$ by $\mathcal{A}$, and let $\mathcal{A}^{*}$ be the dual of $\mathcal{A}$, i.e., the space of continuous linear functionals on $\mathcal{A}$ with the norm

$$
\|\omega\|=\sup _{a \in \mathcal{A}}\{|\omega(a)|:\|a\| \leq 1\}
$$

for every $\omega \in \mathcal{A}^{*}$. Each positive $\omega$ (i.e., such that $\omega\left(a a^{*}\right) \geq 0$ for all $a \in \mathcal{A}$ ) with the unit norm is called a state. The set of all states is convex; the extremal elements of this set are called pure states, the remaining ones - mixed states (for precise definitions see [5], $[16])$. In the case of the commutative algebra $\mathcal{A}=C_{0}(V)$ of continuous functions on a compact space $V$, the states are equivalent to a probability measure on $V$, and one can write

$$
\omega_{\mu}(f)=\int f d \mu
$$

for $f \in C_{0}(V)$. It can be shown that a state $\omega$ is a pure state if and only if it is equivalent to the Dirac measure concentrated on a point $x \in V$; in such a case $\omega_{x}(f)=f(x)$ (see $[15$, p. 70$]$. In other words, the pure states form the space of characters of the algebra $\mathcal{A}$, i.e., the space of ${ }^{*}$-homomorphisms $\omega: \mathcal{A} \rightarrow$ C. It is clear that pure states can be identified with points of $V$, and the algebra $\mathcal{A}$ can be regarded as an algebra of functions defined on the space of characters. 
Let now $\mathcal{A}$ be the non-commutative algebra $C^{*}(\overline{O M})$ and $W \subset \mathcal{A}^{*}$ the space of its pure states. Being non-commutative $\mathcal{A}$ cannot be isomorphic to the algebra $C_{0}(W)$ but, in fact, there is an embedding of $\mathcal{A}=C^{*}(\overline{O M})$ (considered as a vector space) into $C_{0}(W)$,

$$
C^{*}(\overline{O M}) \rightarrow C_{0}(W)
$$

given by $s \mapsto f_{s}$ where $f_{s}(\omega)=\omega(s)$, (see [15, p. 73]). We may regard pure states of a non-commutative algebra as generalizations of the usual concept of point.

If $\mathcal{A}$ is a non-commutative $C^{*}$-algebra, $\pi$ its representation in the Hilbert space $\mathcal{H}$, and $\xi \in \mathcal{H}$, then $a \mapsto(\pi(a) \xi, \xi), a \in \mathcal{A}$, is a positive form on $\mathcal{A}$. This form is a pure state if and only if $\pi$ is a non-zero irreducible representation of $\mathcal{A}$ in $\mathcal{H}$ (see [5]).

Let $\pi_{1}$ and $\pi_{2}$ be two representations of an involutive algebra $\mathcal{A}$ in two Hilbert spaces $\mathcal{H}_{1}$ and $\mathcal{H}_{2}$, respectively. $\pi_{1}$ and $\pi_{2}$ are said to be equivalent representations of $\mathcal{A}$ if there is an isomorphism between $\mathcal{H}_{1}$ and $\mathcal{H}_{2}$ such that $\pi_{1}(a)=\pi_{2}(a)$ up to isomorphism for every $a \in \mathcal{A}$.

Putting the above considerations together we can conclude that pure states or, interchangeably, equivalence classes of irreducible representations of $\mathcal{A}$, can be thought of as non-commutative generalizations of points.

For the sake of concreteness let us focus on the case of the closed Friedman model with the initial and final singularities, remembering that our analysis will be valid, $m u$ tatis mutandis, for other space-times with malicious singularities. The space-time of this model with its b-boundary can be investigated as a single object. The initial and final singularities are two distinct structures given by two representations (strictly speaking by two equivalence classes, each consisting of only one element)

$$
\pi_{p_{i}}: C_{c}^{\infty}\left(\bar{G}, \Omega^{1 / 2}\right) \rightarrow \operatorname{End} L^{2}\left(G_{p_{i}}\right)
$$

$i=1,2$, where $p_{1}$ is the single "limit frame" in the singular fibre over the initial singularity, and $p_{2}$ is the single "limit frame" in the singular fibre over the final singularity. Correspondingly, the two singularities are given by two states (positive forms on $C^{*}(\overline{O M})$ ), $s \mapsto\left(\pi_{p_{i}} \xi, \xi\right), s \in C^{*}(\overline{O M}), \xi \in L^{2}\left(G_{p_{i}}\right), i=1,2$.

If we construct the $C^{*}$-algebra $C^{*}(O M)$ for the space-time of the closed Friedman model without its b-boundary, we must recover the ordinary geometry of this space-time. In fact, it turns out that, in this case, the $C^{*}$-algebra is strongly Morita equivalent to the commutative algebra $C(M)$ of continuous complex functions on $M$.

As strongly Morita equivalent both algebras $C^{*}(O M)$ and $C(M)$ have the same space of equivalence classes of irreducible representations, but in the case of $C^{*}(O M)$ they cannot be identified with points of $M$. However, even in this case they convey an interesting information. For instance, it can be easily seen that the space of equivalence classes of representations $\pi_{q}: C^{*}(O M) \rightarrow \operatorname{End} L^{2}\left(G_{2}\right)$, i.e., the space of states for the closed Friedman model, coincides with the space of space sections $t=$ const. Indeed, because of the maximal symmetry of such constant time sections it is easy to show that two Hilbert spaces $L^{2}\left(G_{p}\right)$ and $L^{2}\left(G_{q}\right)$, where $\operatorname{pr}(p)=x, \operatorname{pr}(q)=y$, such that $x$ and $y$ belong to the same space section, and $p r$ is the usual projection from $O M$ to $M$, are isomorphic, and that this isomorphism induces the transformation of $\pi_{p}(s)$ into $\pi_{q}(s)$ for every $s \in C^{*}(O M)$. It is a nice result since, as is well known, the set of space sections is 
strictly connected with the space of 3 -geometries which serves as the space of states in the canonical quantization of gravity.

Finally, it is interesting to notice that classical singularities in the non-commutative approach seem to know that in the extreme conditions of shrinking mathematical structures familiar from quantum mechanics (algebra of operators on a Hilbert space) give better results than the traditional tools of differential geometry. Is here any lesson which we could learn for our search of the correct theory of quantum gravity? - it remains to be seen.

Acknowledgments. One of the authors (M. H.) expresses his gratitude towards the Belgian Fonds National de la Recherche Scientifique, and the second author (W. S.) towards la Communauté française de Belgique and l'Université de Liège for the financial support during the work on this paper.

\section{References}

[1] R. L. Bishop and S. I. Goldberg, Tensor Analysis on Manifolds, Dover, New York, 1968.

[2] B. Bosshard, On the b-boundary of the closed Friedman model, Commun. Math. Phys. 46 (1976), 263-268.

[3] A. Connes, in: Algèbres d'opérateurs, Lecture Notes in Mathematics, no 725, P. de la Harpe (ed.), Springer, Heidelberg - Berlin - New York 1979.

[4] A. Connes, Noncommutative Geometry, Academic Press, New York, 1994.

[5] J. Dixmier, Les $C^{*}$-algèbres et leur représentations, Gauthier-Villars, Paris, 1969.

[6] J. Gruszczak and M. Heller, Differential structure of space-time and its prolongations to singular boundaries, Intern. J. Theor. Phys. 32 (1993), 625-648.

[7] S. W. Hawking and G. F. R. Ellis, The Large Scale Structure of Space-Time, Cambridge University Press, Cambridge, 1973.

[8] M. Heller, P. Multarzyński, W. Sasin and Z. Żekanowski, On some generalizations of the manifold concept, Acta Cosmologica 18 (1992), 31-44.

[9] M. Heller and W. Sasin, The structure of the b-boundary of space-time, Gen. Rel. Grav. 26 (1994), 797-811.

[10] M. Heller and W. Sasin, Sheaves of Einstein algebras, Int. J. Theor. Phys. 34 (1995), 387-398.

[11] M. Heller and W. Sasin, Structured spaces and their application to relativistic physics, J. Math. Phys. 36 (1995), 3644-3662.

[12] M. Heller, W. Sasin, A. Trafny and Z. Żekanowski, Differential spaces and new aspects of Schmidt's b-boundary of space-time, Acta Cosmologica 18 (1992), 57-75.

[13] R. A. Johnson, The bundle boundary in some special cases, J. Math. Phys. 18 (1977), 898-902.

[14] J. L. Koszul, Fibre bundles and differential geometry, Tata Institute of Fundamental Research, Bombay, 1960.

[15] J. Madore, An Introduction to Noncommutative Differential Geometry and Its Physical Applications, Cambridge University Press, Cambridge, 1995.

[16] G. J. Murphy, $C^{*}$-Algebras and Operator Theory, Academic Press, Boston - New York - London, 1990. 
[17] J. Renault, A groupoid approach to $C^{*}$-algebras, Lecture Notes in Math. 793, Springer, Berlin - Heidelberg - New York, 1980.

[18] W. Sasin, Differential spaces and singularities in differential space-times, Demonstratio Mathematica 24 (1991), 601-634.

[19] W. Sasin and M. Heller, Space-time with b-boundary as a generalized differential space, Acta Cosmologica 19 (1993), 35-44.

[20] B. G. Schmidt, A new definition of singular points in general relativity, Gen. Rel. Grav. 1 (1971), 269-280. 